Nephrologe 2019 $\cdot 14: 505$

https://doi.org/10.1007/s11560-019-00371-z

Online publiziert: 1. Oktober 2019

(C) Der/die Autor(en) 2019

C. S. Haas ${ }^{1}$ M. Nitschke ${ }^{2}$ J. Menne M. M. Guthoff $^{4}$ - A. Gäckler ${ }^{5}$ H. Bruck ${ }^{6} \cdot$ L. Pape $^{7}$.

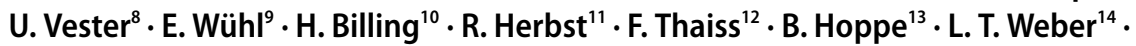
S. Zschiedrich ${ }^{15} \cdot$ T. Feldkamp ${ }^{16} \cdot$ J. Oh $^{12} \cdot$ M. Bald ${ }^{17} \cdot$ B. Schröppel ${ }^{18} \cdot$ J. B. Holle ${ }^{19}$. W. Jabs ${ }^{20} \cdot$ J. Beckermann ${ }^{21} \cdot$ K. Budde ${ }^{22} \cdot$ R. Faulhaber-Walter ${ }^{23} \cdot$ F. Schaefer ${ }^{9}$

'Klinik für Innere Medizin, Nephrologie und Internistische Intensivmedizin, Universitätsklinikum Marburg, Marburg, Deutschland; ${ }^{2}$ Universitätsklinikum Schleswig-Holstein, Campus Lübeck, Lübeck, Deutschland; ${ }^{3}$ MHH Hannover, Hannover, Deutschland; ${ }^{4}$ Medizinische Klinik IV, Sektion Nieren- und Hochdruckkrankheiten, Universitätsklinikum Tübingen, Tübingen, Deutschland; ${ }^{5}$ Universitätsklinikum Essen, Essen, Deutschland; ${ }^{6}$ Medizinische Klinik III - Klinik für Innere Medizin, Nephrologie, Diabetologie, Rheumatologie, Endokrinologie, Helios Klinikum und KfH Nierenzentrum Krefeld, Krefeld, Deutschland; ${ }^{7}$ Kinderklinik, MHH Hannover, Hannover, Deutschland; ${ }^{8}$ Kindernephrologie, Helios St Johannes, Duisburg, Deutschland; ${ }^{9}$ Zentrum für Kinder- und Jugendmedizin, Universitätsklinikum Heidelberg, Heidelberg, Deutschland; ${ }^{10}$ Universitätsklinikum Tübingen Kinderklinik, Tübingen, Deutschland; ${ }^{11}$ Klinikum Chemnitz gGmbH, Chemnitz, Deutschland; ${ }^{12} \mathrm{III}$. Medizinische Klinik und Poliklinik, Universitätsklinikum Hamburg-Eppendorf, Hamburg, Deutschland; ${ }^{13}$ Uniklinikum Bonn, Bonn, Deutschland; ${ }^{14} \mathrm{Klinik}$ und Poliklinik für Kinder- und Jugendmedizin, Medizinische Fakultät und Uniklinik Köln, Universität zu Köln, Köln, Deutschland; ${ }^{15}$ Klinik für Innere Medizin IV, Nephrologie und Allgemeinmedizin, Universitätsklinikum Freiburg, Medizinische Fakultät, Albert-Ludwigs-Universität Freiburg, Freiburg, Deutschland; ${ }^{16}$ Campus Kiel, Universitätsklinikum Schleswig-Holstein, Kiel, Deutschland; ${ }^{17}$ Olgahospital, Klinikum Stuttgart, Stuttgart, Deutschland; ${ }^{18}$ Universitätsklinikum Ulm, Ulm, Deutschland; ${ }^{19}$ Klinik für Pädiatrie m.S. Gastroenterologie, Nephrologie und Stoffwechselmedizin, Charité - Universitätsmedizin Berlin, Berlin, Deutschland; ${ }^{20}$ Vivantes Klinikum im Friedrichshain, Berlin, Berlin, Deutschland; ${ }^{21}$ St. Marienhospital Vechta, Vechta, Deutschland; ${ }^{22}$ Charité Berlin, Berlin, Deutschland; ${ }^{23}$ Facharztzentrum Aarberg, Aarberg, Deutschland

\title{
Erratum zu: Charakterisierung von Patienten mit atypischem hämolytisch-urämischen Syndrom (aHUS) in Deutschland
}

\section{Daten aus dem globalen aHUS-Register}

\section{Erratum zu:}

Nephrologe 2019

https://doi.org/10.1007/s11560-019-

0357-4

Im ursprünglich veröffentlichten Beitrag (online) fehlten der Interessenkonflikt und einige Abbildungen. In der Onlineund der Print-Version des Beitrages wurden der Interessenkonflikt und die Abbildungen eingefügt. Online und Print-Version sind die korrekten, korrigierten Versionen des Beitrages. Wir bitten Sie, den Fehler zu entschuldigen.

\author{
Korrespondenzadresse \\ Prof. Dr. F. Schaefer \\ Zentrum für Kinder- und Jugendmedizin, \\ Universitätsklinikum Heidelberg \\ Im Neuenheimer Feld 430, 69120 Heidelberg, \\ Deutschland \\ franz_schaefer@med.uni-heidelberg.de
}

Open Access. Dieser Artikel wird unter der Creative Commons Namensnennung 4.0 International Lizenz (http://creativecommons.org/licenses/by/4.0/deed. de) veröffentlicht, welche die Nutzung, Vervielfältigung, Bearbeitung, Verbreitung und Wiedergabe in jeglichem Medium und Format erlaubt, sofern Sie den/die ursprünglichen Autor(en) und die Quelle ordnungsgemäßnennen, einen Link zur Creative Commons Lizenz beifügen und angeben, ob Änderungen vorgenommen wurden.

Die Online-Version des Originalartikels ist unter https://doi.org/10.1007/s11560-019-0357-4zu finden 